Revista de la Facultad de Ingeniería Industrial

18(1): 53-67 (2015) UNMSM

ISSN: 1560-9146 (Impreso) / ISSN: 1810-9993 (Electrónico)

\title{
Análisis y evaluación del capital intelectual en una universidad pública del Perú
}

\section{RESUMEN}

El crecimiento del capital intelectual es un rubro tan importante en una institución universitaria. La falencia de ella debe enfrentarse mediante acciones estratégicas. Facilitar la creación de nuevo conocimiento, apunta a la innovación a través de la reutilización y apoyo de la habilidad de la gente por medio de organizaciones para lograr un mejor desempeño en la empresa.

El capital humano constituye la fuente de innovación y renovación de las universidades, y se encuentra en: las ideas geniales que aparecen en el laboratorio, el conocimiento generado a través del proceso de investigación, las experiencias obtenidas en el desempeño laboral, o en las habilidades adquiridas por el personal mediante un proceso de desarrollo organizacional. Es imperativo gestionar el conocimiento a través de una concepción diferente respecto al capital humano, que como uno de los ejes del capital intelectual se convierten en actores dinamizadores de la gestión y socios estratégicos de la operación para ser cada vez más eficientes y eficaces a través de la racionalidad de los recursos que son cada vez más escasos.

Palabras clave: capital intelectual, capital humano, conocimiento generado, desarrollo organizacional

ANALYSIS AND EVALUATION OF UNIVERSITY INTELLECTUAL CAPITAL OF PERU PUBLIC

\section{ABSTRACT}

The growth of intellectual capital, is an important item in a university institution. The failure of this heading must face through strategic actions. Facilitate the creation of new knowledge, underpins innovation through reuse and support of the people through organizations ability to achieve a better performance in the company.

Human capital is the source of innovation and renewal of universities, and is located in: great ideas that appear in the laboratory, the knowledge generated through the research process, the experiences obtained in the work performance, or the skills acquired by staff through a process of organizational development. It is imperative to manage knowledge through a different conception regarding human capital, which, as one of the axes of intellectual capital, become actors facilitators of management and strategic partners of the operation to be increasingly more efficient and effective through the rationality of resources that are increasingly scarce.

Keywords: human capital, intellectual capital, knowledge, organizational development

\section{PLANTEAMIENTO DEL PROBLEMA}

Actualmente el estudio del capital intelectual en las universidades públicas del Perú es un tema relativamente nuevo.

La Facultad de Ingeniería Industrial de la UNMSM ha superado un proceso de acreditación; sin embargo, no existe una forma de medición del capital intelectual; esto trae como consecuencia no conocer a detalle factores influyentes, ni mucho menos gestionarlos.

Asimismo, al no poder gestionar el capital intelectual de la Facultad, no se pueden tomar medidas adecuadas que orienten el crecimiento de este rubro tan importante en una institución universitaria. Tampoco existe un estudio sobre su comportamiento, lo cual constituye una falencia que debe enfrentarse mediante acciones estratégicas.

De seguir el escenario tal y como se presenta actualmente, la Facultad de Ingeniería Industrial podría perder el sitial logrado, así como la acreditación lograda; para superar este problema sería necesario realizar un estudio actualizado acerca de sus componentes y comportamiento, con el fin de poder formular estrategias de accionar para acrecentar su valor. Sin embargo en la actualidad no se lleva a cabo.

\section{Formulación del problema}

\section{Problema general}

¿Cómo medir y controlar el Capital Intelectual en la Facultad de Ingeniería Industrial de la UNMSM?

\section{Problemas específicos}

a) ¿Qué indicadores de Capital Intelectual son los adecuados para la Facultad de Ingeniería Industrial de la UNMSM?

b) ¿Cómo organizar la información dispersa sobre Capital Intelectual en la Facultad de Ingeniería Industrial de la UNMSM?

c) ¿Cómo determinar la evolución del Capital Intelectual en la Facultad de Ingeniería Industrial de la UNMSM?

Magíster en Ingeniería Industrial, profesor del Departamento de Diseño y Tecnología IndustrialUNMSM. E-mail: jyenque@yahoo.es 


\section{OBJETIVOS DE LA INVESTIGACIÓN}

\section{Objetivo general}

Diseñar un modelo que permita medir y controlar del capital intelectual en la Facultad de Ingeniería Industrial de la UNMSM.

\section{Objetivos específicos}

a) Determinar los indicadores de Capital Intelectual adecuados para la Facultad de Ingeniería Industrial de la UNMSM

b) Diseñar un modelo que permita organizar el Capital Intelectual en la Facultad de Ingeniería Industrial de la UNMSM

c) Diseñar un modelo que permita analizar la evolución del capital intelectual en la Facultad de Ingeniería Industrial de la UNMSM

\section{ANTECEDENTES DEL PROBLEMA}

En el proyecto multidisciplinario de investigación desarrollado por Inche J. \& Chung (2004), "Gestión del conocimiento para una universidad competitiva"; se plantea ¿Cómo medir y controlar el Capital Intelectual de una Universidad Nacional?; para ello formulan, validan y aplican un modelo de Gestión del Conocimiento unido a un tablero de mando integral. Para ello, se reformula las perspectivas clásicas del Tablero de mando (BSC) adaptado a la realidad de una universidad orientada a la competitividad. El resultado de este análisis se presenta en la figura 1.

Figura 1.

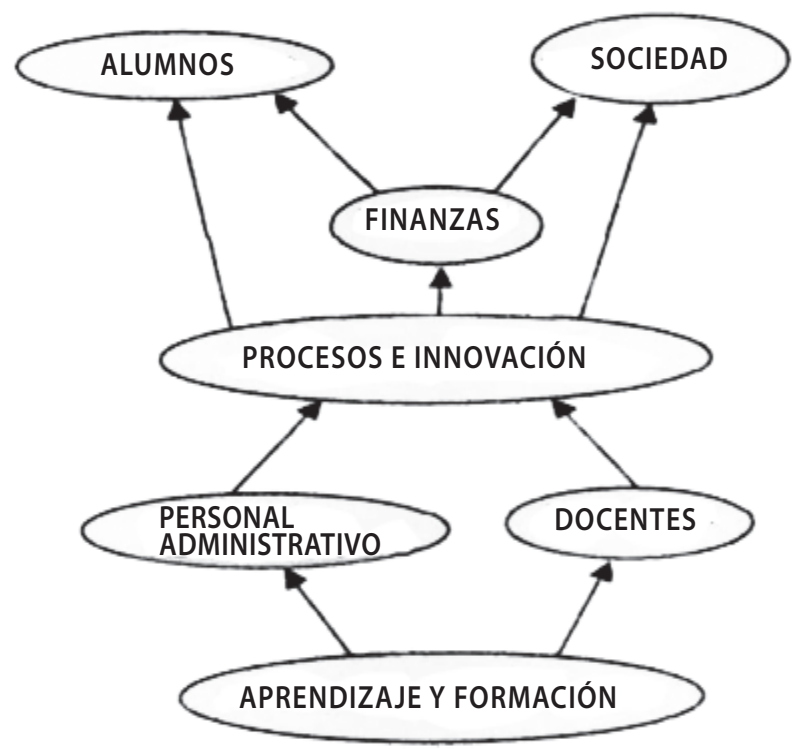

Fuente: Inche J. \& Chung A. (2004).
Como se aprecia en la figura el fin primordial del sistema no es beneficio económico de los accionistas sociedad; este cambio se traduce en los objetivos del sistema.

Por otro lado; (Inche Mitma, 2010) en su trabajo de investigación "Modelo dinámico de gestión del conocimiento basado en el aprendizaje organizacional en una institución educativa en el Perú", profundiza conceptos sobre la aplicación de la gestión del conocimiento en una institución educativa en el Perú; asimismo propone un modelo de Gestión de Conocimiento (GESCON), validado en la UNMSM como parte de la tesis.

Donde se evidencia que tanto el capital intelectual como sus elementos tienen un valor positivo en el aprendizaje organizacional el cual se traduce en la generación de almacenes y flujo de conocimiento.

Por otro lado, (García, 2010) en su tesis "Diseño de un modelo multifactorial y dinámico (MFD) para la medición del capital intelectual de las empresas de manufactura del Perú."; propone un modelo multifactorial y dinámico (MFD) el cual permite la medición del capital intelectual en las empresas de manufactura; para la validación de dicho modelo, se hizo la prueba respectiva en una empresa real, a fin de analizar las consecuencias de su aplicación.

Un aspecto importante que señala esta investigación es que la contabilidad tradicional no está preparada para la medición del capital intelectual de una empresa, esto se agrava más aún si se considera la importancia gravitante en diferentes aspectos de la organización.

\section{Bases teóricas}

\section{El Capital Intelectual}

Cuando las compañías descubren que los estados financieros no reflejar técnicamente variables alternas que generen valor y no se identifican en los balances, se evidencia, en la retina de los empresarios, unidades como la calidad, el prestigio y el cumplimiento, entre otros. Estos elementos son tratados por el economista Kenneth Galbraith, quien en 1969, los denomina: Capital Intelectual (C.I), definiéndolo como el mecanismo generador de plus valor entre el valor de la empresa y el valor técnicamente contable (libros).

Para afianzar lo expuesto y complementar la discusión, se citan algunas corrientes y autores que reafirman el aporte del Capital Intelectual a la Gestión del Conocimiento, organizados en dos grandes apartados: en primera instancia, se destacan autores como (Von Roth, 2008) y (Diaz Muñante, 2007), quienes resaltan la existencia del 
C.I en el contexto de la Gestión del Conocimiento (G.C). La autora (Von Roth, 2008) define la Gestión del Conocimiento como "un conjunto de disciplinas de administración que trata el Capital Intelectual como un activo de la empresa".

Entre tanto, para (Diaz Muñante, 2007), el C.I "es la agrupación de activos que una sociedad posee que, a pesar de no estar discriminados en un estado contable son generadores de valor". De acuerdo con estas definiciones, se percibe claramente, que la meta principal de la G.C. es entregar la capacidad intelectual de la empresa a los trabajadores del conocimiento; lo cual implica abarcar diversas fuentes de conocimiento y cultivarlo en ambientes donde éste reside para darle valor agregado a las compañías.

Es pertinente hacer alusión al aporte conceptual hecho por el profesor Bueno (2001) definiendo el C.I como "el conjunto de capacidades, destrezas y conocimientos de las personas que generan valor ya sea para las comunidades científicas, las universidades, las organizaciones y la sociedad en general".

(Bueno Campos, 2003) se refiere a "la percepción conceptual de Sveiby (1997), y Stewart (1997) donde manifiesta que el capital "invisible" se convierte en riqueza para las empresas y las naciones, reflejado en dos dimensiones: la estática (valor del intangible en un determinado periodo de tiempo) y la dinámica (acciones basadas en aplicaciones del intelecto, tales como adquirir, aumentar y controlar."

\section{La Gestión del Conocimiento}

Este concepto (del inglés Knowledge Management) es aplicado en las organizaciones. A fin de transferir el conocimiento desde el lugar dónde se genera hasta el lugar en dónde se va a emplear (Fuentes Morales, 2009), e implica el desarrollo de las competencias necesarias al interior de las organizaciones para compartirlo y utilizarlo entre sus miembros, así como para valorarlo y asimilarlo si se encuentra en el exterior de estas.

La gestión del conocimiento es el área dedicada a la dirección de las tácticas y estrategias requeridas para la administración de los recursos humanos intangibles en una organización (Brookin, 2010) (Brooking, 1996).

Sus perspectivas tácticas y operativas, se detallan en la gestión del capital intelectual y se centra en la forma conocer y administrar las actividades relacionadas con el conocimiento como su creación, captura, transformación y uso. Su función es planificar, implementar y controlar todas las actividades relacionadas con el conocimiento y los programas requeridos para la administración efectiva del capital intelectual (Wiig) .

\section{Técnicas de Gestión del Conocimiento}

El proceso implica técnicas para capturar, organizar, almacenar el conocimiento de los trabajadores, para transformarlo en un activo intelectual con beneficios compartibles.

Actualmente, las tecnologías de información permiten contar con herramientas que apoyan la gestión del conocimiento en las empresas, apoyando en la recolección, la transferencia, la seguridad y la administración sistemática de la información, junto con los sistemas diseñados para ayudar a hacer el mejor uso de ese conocimiento.

Es un mercado del software y un área en la práctica de la consultoría, relacionada a disciplinas tales como inteligencia competitiva. Un tema particular de la administración del conocimiento es que el conocimiento no se puede codificar fácilmente en forma digital, tal como la intuición de los individuos dominantes que viene con años de experiencia y de poder reconocer los diversos patrones del comportamiento que alguien con menos experiencia no puede reconocer.

El proceso de la Administración del Conocimiento, también conocido en sus fases de desarrollo como "aprendizaje corporativo" o "aprendizaje organizacional", tiene los siguientes objetivos:

- Identificar, recabar y organizar el conocimiento existente.

- Facilitar la creación de nuevo conocimiento.

- Apuntalar la innovación a través de la reutilización y apoyo de la habilidad de la gente a través de organizaciones para lograr un mejor desempeño en la empresa.

La transferencia del conocimiento (un aspecto de la Administración del Conocimiento) ha existido siempre como proceso en las organizaciones. De manera informal por medio de las discusiones, sesiones, reuniones de reflexión, etc., y de manera formal por medio del aprendizaje, el entrenamiento profesional y los programas de capacitación.

Los profesionales de la Gestión del Conocimiento pueden utilizar un léxico específico para explicar la dinámica de la transferencia del conocimiento. Por ejemplo en los diez años pasados, Internet ha visto a grupos establecer discusiones sobre el uso del capital intelectual como valor métrico; el significado tácito contra conocimiento explícito o más. 


\section{El Modelo del Balanced Scorecard (BSC)}

Elaborado y desarrollado en 1992, por Kaplan y Norton (2000), es uno de los trabajos pioneros en materia de Capital Intelectual; su análisis incluye indicadores de gestión excluidos, haciendo de este compendio, una herramienta para la toma de decisiones.

El modelo del BSC presenta cuatro perspectivas estipuladas de la siguiente manera:

a) La perspectiva financiera: referida a los indicadores financieros, flujos de caja, análisis de rentabilidad de cliente y producto, gestión de riesgo,

b) La perspectiva de cliente: identifica los valores relacionados con los clientes,

c) La perspectiva de procesos internos: satisfacción de clientes internos y clientes externos, por medio, tanto de procesos de innovaciones, de operaciones, como de servicio posventa,

d) La perspectiva del aprendizaje: se refiere a innovación, crecimiento, motivación y capacidad de aprender y crecer con base en la gestión de los empleados.

\section{Necesidad de Gestionar el Capital Intelectual en las Universidades}

Las universidades son productoras de conocimiento per ser, incorporando en resultados de investigación, publicaciones, estudiantes formados y relaciones productivas con sus stakeholders. Entre sus recursos más valiosos están sus investigadores, gestores, estudiantes y redes de relaciones. Recursos que son parte de su capital intelectual, y a pesar de su importancia, rara vez son tratados de manera específica.

Otras razones que justifican la importancia y necesidad de establecer un modelo de valoración, gestión y difusión del capital intelectual en la universidad, es el hecho de que existe una creciente de mayor información y transparencia sobre el uso de los fondos públicos (Ramírez Córcoles), con lo que las universidades públicas se ven forzadas a difundir más información a sus stakeholders.

El capital intelectual se representa generalmente como formado por tres componentes básicos y fuertemente interrelacionados: capital humano, capital estructural y capital relacional; Stewart, 1997; Edvinsson y Malone, 1997; Euroforum, 1998; CIC, 2002, etc.).

Paralasuniversidades, definimosestoscomponentes como sigue: Capital Humano: El conjunto de conocimiento explícito y tácito del personal de la universidad (profesores, investigadores y personal de administración y servicios) adquirido a través de una educación formal e informal y de procesos de actualización incluidos en sus actividades. El capital humano constituye la fuente de innovación y renovación de las universidades, y se encuentra en: las ideas geniales que aparecen en el laboratorio, el conocimiento generado a través del proceso de investigación, las experiencias obtenidas en el desempeño laboral, o en las habilidades adquiridas por el personal mediante un proceso de desarrollo organizacional. Capital Estructural: El conocimiento explícito relativo al proceso interno de difusión, comunicación y gestión del conocimiento científico y técnico en la universidad. Es la capacidad organizacional, la infraestructura tecnológica, física o comunicacional que mantiene, desarrolla y sostiene el capital humano. Se puede dividir en Capital Organizacional (el entorno operativo derivado de la interacción entre investigación, gestión y procesos de organización, tecnología y cultura) y Capital Tecnológico (patentes, licencias, software, bases de datos, etc.). Indicadores representativos para el capital estructural son las inversiones en tecnología de información y literatura (en unidades monetarias).

Capital Relacional: Recoge el amplio conjunto de relaciones económicas, políticas e institucionales desarrolladas y mantenidas por las universidades, y también recoge cómo la universidad es percibida: su imagen, atractivo, fiabilidad, etc. Esta categoría podría ser asimilada con la conocida Tercera Misión de las Universidades, que incluye todas las actividades y relaciones entre la universidad y los socios no académicos: firmas, organizaciones no lucrativas, autoridades públicas, gobierno local y sociedad en general. Indicadores típicos son el número de miembros en asociaciones científicas, vínculos con otras universidades, y contactos con alumnos. Sin embargo, el capital intelectual es más que la simple suma de estos tres elementos, su riqueza reside en las interconexiones entre ellos. Capaz de generar un incremento en el valor de la universidad, su propósito es permitir tomar ventaja de oportunidades frente a sus competidores.

El Balanced Scorecard como Herramienta de Gestión del Capital Intelectual en las Universidades

Introducido como una herramienta dirigida a las organizaciones empresariales, el BSC ha encontrado considerable apoyo y ha sido usado en la valoración estratégica de instituciones de educación superior. 
La Universidad está inmersa en un entorno de complejidad creciente por la necesidad de mejora continua en eficacia, eficiencia y economía. Como institución con una responsabilidad social importante de administrar adecuadamente los recursos que posee, ya que su mal uso puede afectar a la calidad de la educación (López y García, 2007). Ante este panorama, el BSC constituye un enfoque realmente innovador que enriquece el control de la gestión de los intangibles de las universidades.

La elección del Cuadro de Mando Integral como sistema de gestión de intangibles en las universidades se debe a que, hoy por hoy, es considerado como una de las herramientas más eficaces para implantar y hacer operativa la cultura de la calidad y el incremento del rendimiento y que ha sido implementada con éxito en algunas universidades (por ejemplo, University of Edinburg, the Open University, Glasgow Caledonian University, Napier University, University of California, 1999 (O'Neil et al., 1999); Ohio State University, 2007; Universitat Pompeu Fabra, 2000; Universitat Jaume I, 2000; The Fort Hays State University, 2003; Zbinden, 2002, etc.).

Entre los principales beneficios de la implantación del BSC en las universidades se basa en un conjunto de indicadores no solo financieros sino la misión y los objetivos de la universidad en su totalidad. Entre las principales aportaciones del BSC a la universidad cabe destacar las siguientes (Boned y Bagur, 2007; Universidad de Cádiz, 2005, p. 8-10); EI BSC pretende traducir las estrategias de la Institución en una serie de indicadores que informen de la consecución de los objetivos y de las causas que provocan los resultados obtenidos.

Ayudará a identificar y alinear los objetivos y el trabajo de todos con los fines de la Institución.

Se utilizará como un simulador, al determinar las relaciones causa-efecto entre las estrategias e indicadores, permitiendo identificar las acciones de mejora. Por último, con el BSC se logra la interrelación entre todas las estrategias; por tanto, aquellas definidas para la consecución de un objetivo concreto para la Institución, estarán contribuyendo, irremediablemente, a la consecución de otros distintos.

La aplicación del BSC en las universidades, al igual que en el resto de organizaciones, se deriva y construye partiendo de la visión y estrategia de la institución y está compuesto por un conjunto de indicadores financieros y no financieros relativos a los factores que determinan su éxito. En la elaboración de un BSC es necesario que la estrategia esté definida. Con este fin, un importante paso de inicio es el desarrollo de un Plan Estratégico institucional que recoja los objetivos y estrategias a largo plazo, así como las líneas de trabajo. La construcción del modelo se hace de tal forma que los indicadores diseñados para cada caso particular, mantengan entre ellos una fuerte conexión, planteada en forma de relaciones de causa-efecto a lo largo de la cadena de valor objeto de análisis. Tales indicadores pueden ser de tipo cualitativo (encuestas de opinión realizadas al personal docente e investigador, al personal de administración, a los alumnos, a las empresas, etc.) o cuantitativo, pudiendo ser éstos monetarios (como son el coste de un programa por alumno o por crédito docente, complemento medio de docencia por profesor) o no monetarios (porcentaje de eficiencia terminal por programa, tiempo medio por alumno en finalizar una titulación, etc.).

Una vez definido el conjunto de indicadores para cada perspectiva, se registran con valores históricos, se comparan con una meta o indicador objetivo y con el valor que resulte de las mejores prácticas (benchmarking). Para adecuar el BSC al ámbito de las universidades, O'Neil y Harold (1999) realizaron una serie de modificaciones: pasar del "balanced scorecard" a un "academia scorecard", rediseñando dos perspectivas para adaptarlas a la Universidad. La "perspectiva financiera" fue cambiada por "perspectiva de la administración académica", modificando la pregunta: "cómo nos ven nuestros accionistas" por: "cómo nos ven los líderes universitarios".

EI BSC sugiere que la organización sea vista desde cuatro perspectivas, desarrollando métricas, recogiendo datos y analizándolos para cada una de esas perspectivas.

\section{Estas perspectivas claves son:}

Perspectiva de aprendizaje y crecimiento: Esta perspectiva recoge la habilidad de los empleados, la calidad de los sistemas de información y los efectos de la coordinación de los procedimientos y rutinas de la organización en el logro de una actuación o desempeño que represente un gran adelanto. Los procesos solamente tendrán éxito si son conducidos por empleados formados y motivados, provistos con información precisa y a tiempo. Factores que contribuyen a un alto rendimiento, el clima del lugar de trabajo, la moral de los empleados, estrategias profesionales de desempeño, uso efectivo de la tecnología, etc. En la Universidad se podría enfatizar en esta perspectiva la capacidad de la organización de sostener e incrementar la habilidad de satisfacer las necesidades del cliente y mejorar los procesos de eficacia y eficiencia, que siguiendo a Bayley et 
al. (1999) se puede agrupar en: a) Enseñanza; b) Calidad de las instalaciones para los alumnos; c) Procesos de conducción de la misión y sistemas de recompensa.

Perspectiva de los procesos internos: Esta perspectiva se centra en objetivos e indicadores que informen sobre la mejora de los procesos internos que conducen al éxito financiero y al logro de clientes satisfechos. Para alcanzar los objetivos organizacionales y las expectativas de los clientes, las organizaciones deben identificar los procesos claves en los cuales se debe sobresalir. Los procesos internos son los mecanismos a través de los cuales las expectativas de rendimiento son alcanzadas. Entre los objetivos de la perspectiva interna en una Universidad se incluye la excelencia en la enseñanza y aprendizaje, excelencia en una malla curricular innovadora, proveer a los profesores un ambiente de trabajo que facilite el cumplimiento de sus metas, etc.

Perspectiva del cliente: Se recogen los objetivos e indicadores relacionados con la ampliación de nuestro ámbito de actuación, fidelidad de los usuarios a nuestros servicios, aumento de satisfacción de los mismos, la mejora de la imagen de la institución, así como las relaciones y las comunicaciones con su entorno.

Perspectiva financiera: Incluiría los objetivos e indicadores relacionados con la mejora de la situación financiera de la Universidad. Para las organizaciones públicas, las consideraciones financieras raramente serán el objetivo principal. El éxito de las organizaciones públicas debería ser medido a través de cuán eficaz y eficientemente cumplen las necesidades de sus clientes. Así pues, aquí no se trata de maximizar el beneficio, sino de prestar los servicios con calidad, eficacia y empleando el menor volumen de recursos.

Un conjunto de medidas financieras claves, tales como el ratio de operaciones netas, estado de recursos, ratio de reinversión, competitividad de investigación y capacidad deudora, valoran la riqueza financiera de la universidad. En el nivel operativo, los objetivos y medidas financieras se centran en la ecuación coste/calidad de los servicios y en una reducción de los costes o coste de anulación de estrategias dentro de áreas específicas del negocio. Así pues, en esta perspectiva se pueden identificar fundamentalmente tres aspectos: a) Las fuentes de financiación de la institución universitaria, b) Cómo asignar los recursos a las distintas unidades orgánicas, es decir, las decisiones de financiación e inversión, y c) La mejora de la eficiencia.

\section{METODOLOGÍA}

\section{Diseño de la Investigación}

El tipo de investigación a desarrollar es descriptiva y explicativa por qué se va a analizar como es y cómo se manifiesta determinado modelo de medición, los estudios descriptivos buscan especificar las propiedades importantes de personas, instituciones, empresas, grupos, comunidades o cualquier otro fenómeno que sea sometido a analizar, medir o evaluar diversos aspectos, dimensiones o componentes del fenómeno o fenómenos a investigar; desde el punto científico, describir es medir. Al emplear la estadística descriptiva como método, se parte del hecho de que hay una cierta realidad (o sector del mundo) que resulta insuficientemente conocida $\mathrm{y}$, al mismo tiempo, relevante e interesante para ciertos desarrollos.

\section{Hipótesis general y secundaria}

Como hipótesis general se planteó lo siguiente:

Mediante un modelo basado en el cuadro de mando integral (CMI) se logrará medir y controlar adecuadamente el capital intelectual de la FII UNMSM.

Como hipótesis secundarias se consideraron las siguientes:

a) Los indicadores basados en un modelo de CMI son los adecuados para medir el capital intelectual en la FII - UNMSM

b) Mediante un modelo basado en el CMI permitirá organizar la información dispersa del capital intelectual en la FII - UNMSM

c) La aplicación de un análisis basado en el modelo del CMI permitirá determinar la evolución del Capital Intelectual en la FII - UNMSM

\section{MARCO CONTEXTUAL}

\section{Características de la Facultad de Ingeniería Industrial}

\section{Reseña histórica}

En 1957 la UNMSM, a fin de investigar los problemas humanos más urgentes planteados por la realidad económica y social del país, crea el Instituto de Relaciones Humanas y Productividad, para formar expertos en: Personal, Administración, Análisis de Costos y Mercado, y Relaciones de Trabajo.

El año 1960 el Instituto se convierte en Escuela Superior de Graduados de la Facultad de Ciencias Económicas, a la que acceden quienes, con estudios superiores concluidos, deseaban ser 
expertos en: Relaciones Públicas y Publicidad, Relaciones Industriales, e Ingeniería Industrial de Producción y Gerencia.

En octubre de 1965 se crea la Escuela de Relaciones Industriales y Productividad (ERIP) en reemplazo de la anterior Escuela Superior. Se ofrecen tres carreras profesionales de ingeniería para egresados de educación secundaria: Ingeniería de Racionalización y Productividad, Ingeniería de Programación e Ingeniería de Relaciones Humanas.

El 18 de febrero de 1969 se promulga el Decreto Ley N¹7437, Ley Orgánica de la Universidad Peruana, que dispone la reforma del sistema universitario. La reestructuración realizada en San Marcos, lleva a fusionar en la carrera de Ingeniería Industrial, las tres carreras de Ingeniería que ofrecía la ERIP, constituyendo la unidad académica denominada Programa Académico de Ingeniería Industrial.

En diciembre de 1983 se da la Ley Universitaria $N^{\circ}$ 23733. En cumplimiento de ella, la Asamblea Estatutaria de la UNMSM, en septiembre de 1984, promulga el Estatuto de la Universidad, cuyo Art. $38^{\circ}$ crea la Facultad de Ingeniería Industrial e Ingeniería Electrónica y dentro de ella la Escuela Académico Profesional de Ingeniería Industrial.

Finalmente, el 7 de diciembre de 1988, la Asamblea Universitaria crea la FACULTAD DE INGENIERÍA INDUSTRIAL, la misma que luego de más de veintiún años ha consolidado su organización, y ha creado la Escuela Académico Profesional de Ingeniería Textil y Confecciones, con la permanente responsabilidad de cumplir a cabalidad con el rol que le corresponde en el desarrollo del país.

\section{Misión}

"facultad con alto nivel académico en la formación de profesionales en el ámbito de la Ingeniería Industrial, flexibles, innovadores y capaces de responder a las necesidades de la sociedad peruana.

\section{Visión}

"Ser la mejor facultad de Ingeniería Industrial del sistema universitario peruano reconocida internacionalmente por formar profesionales líderes".

\section{Gestión del Talento Humano en la Facultad de Ingeniería Industrial}

"La Gestión del talento humano de la Facultad de Ingeniería Industrial de la Universidad Nacional Mayor de San Marcos, se ha caracterizado por poseer potencialidades que desarrolla en beneficio propio y del entorno relacionado con procesos que brindan servicio eficiente, eficaz y efectivos. Dentro de este marco, y para promover el conocimiento de las herramientas con las que la facultad direcciona el macro proceso de Gestión del Talento Humano radica en detectar, apoyar, e impulsar, poner a prueba e incentivar el talento de los funcionarios para lograr transformaciones. Situación que se muestra mediante el documento denominado.

"Lineamientos y Directrices Generales de la Gestión del Talento Humano de la Facultad de Ingeniería Industrial de la Universidad Nacional Mayor de San Marcos", y cuyo objetivo principal es divulgar a la comunidad universitaria las orientaciones generales que determinan el quehacer de dicha Gestión, en pro del cumplimiento de la misión, visión y los objetivos institucionales.

Figura 2. Macro procesos de la unidad de personal

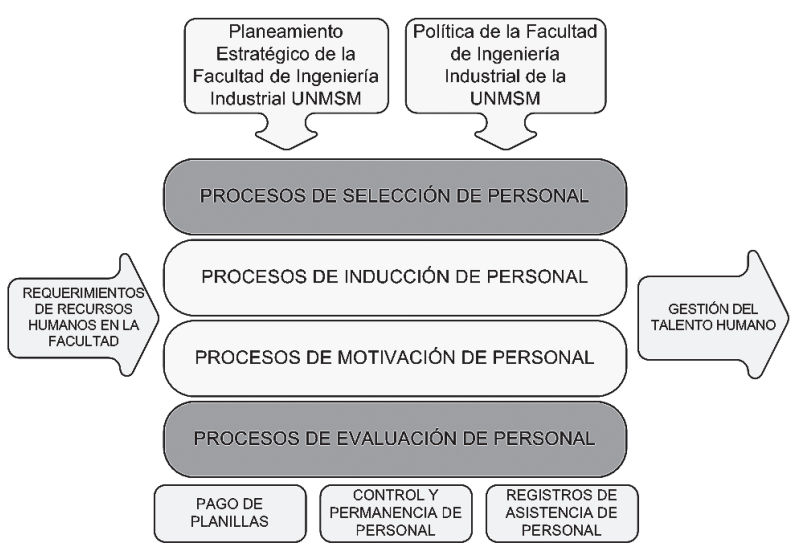

Fuente: Elaboración propia.

\section{Principios Rectores de la Gestión de Talento Humano}

Los principios rectores deGestión del talento en la Unidad de Personal de la Facultad de Ingeniería Industrial, son los siguientes:

Objetivos Estratégicos de Gestión y Relaciones Institucionales del Plan Estratégico Institucional de la Facultad de Ingeniería Industrial 2012 - 2021.

* Optimizar los sistemas de gestión organizacional

* Optimizar la gestión de los recursos humanos

* Mejorar la relación organizacional de la Facultad.

* Mejorar el servicio de atención al cliente interno como al externo 


\section{Objetivos Estratégicos de Calidad}

- Desarrollar e implementar el Sistema de Calidad de la Facultad aplicando el enfoque sistémico y de gestión de procesos.

- Implementar y desarrollar el Sistema de Evaluación de la Calidad Universitaria de la Facultad para lograr una Acreditación de la Calidad.
- Fomentar y diseminar una cultura de evaluación, planeación, innovación y mejora continua en los miembros de la Facultad.

\section{Procesos de Gestión del Talento Humano - Docentes y Administrativos}

En la siguiente tabla se consolidan los procesos y directrices generales de la GTH en la Facultad de Ingeniería Industrial de la Universidad Nacional Mayor de San Marcos mediante las cuales se gestiona el capital humano.

Tabla 1. Procesos y Directrices Generales de la Gestión de Talento Humano en la Facultad de Ingeniería Industrial

\begin{tabular}{|c|c|c|}
\hline PROCESO & DEFINICIÓN & SUBPROCESO \\
\hline \multirow{3}{*}{ Selección de personal } & \multirow{3}{*}{$\begin{array}{l}\text { Proceso por el cual se selecciona al mejor } \\
\text { recurso disponible, y se le integra a la } \\
\text { institución, generando un aporte de capital } \\
\text { humano. }\end{array}$} & Análisis y descripción de puestos de trabajo \\
\hline & & Selección de personal \\
\hline & & Vinculación de personal \\
\hline \multirow{4}{*}{ Inducción de personal } & \multirow{4}{*}{$\begin{array}{l}\text { Se transmite la cultura organizacional, } \\
\text { integrándolo a la institución, generando } \\
\text { la sinergia necesaria para el desarrollo de } \\
\text { funciones, actividades y tareas. }\end{array}$} & Docentes Inducidos \\
\hline & & Administrativos Inducidos \\
\hline & & Entrega de Manuales \\
\hline & & Presentación de personal. \\
\hline \multirow{3}{*}{ Motivación de Personal } & \multirow{3}{*}{$\begin{array}{l}\text { Proceso de mejora de competencias y } \\
\text { habilidades en el Personal docente y } \\
\text { administrativo de la Facultad. }\end{array}$} & Programa de Capacitación \\
\hline & & Registro de los procesos de capacitación \\
\hline & & Programa de Becas e incentivos \\
\hline \multirow{3}{*}{ Evaluación de Personal } & \multirow{3}{*}{$\begin{array}{l}\text { Proceso por el cual se evalúa el ingreso, } \\
\text { ratificación y promoción docente }\end{array}$} & Programa de Ingreso a la carrera docente \\
\hline & & Programa de Ratificación \\
\hline & & Programa de promoción \\
\hline
\end{tabular}

\section{Políticas de Gestión del Talento Humano}

La Unidad de Personal de la Facultad, tiene como políticas las siguientes:

1. Respeto por el individuo: Basado en la participación del mismo en todos los procesos fundamentales de la institución.

2. Respeto de los derechos humanos y constitucionales: El respeto de todos los colaboradores, como personas y respetuosos de sus derechos fundamentales.

3. Igualdad de condiciones para los colaboradores: Trato justo e igualitario para todos los colaboradores de la institución.

4. Desarrollo de las personas y de la institución: fomento del desarrollo personal a través del plan estratégico individual y coerción con el desarrollo de la institución.

5. Seguridad y salud en el trabajo: fomentar una cultura de seguridad y salud en el trabajo.

\section{ANÁLISIS E INTERPRETACION DE RESULTA- DOS}

Estado actual del sistema de gestión del capital intelectual en el personal de la Facultad de Ingeniería Industrial de la Universidad Nacional Mayor de San Marcos

Con la finalidad de darle respuesta planteamientos propuestos se identifica cuáles son los componentes de gestión que realiza la universidad en sus lineamientos de la gestión del talento Humano. 
Faculta de Ingeniería Industrial de la Universidad Nacional Mayor de San Marcos.

Funciones de la gestión del talento humano en la facultad de Ingeniería industrial

\begin{tabular}{|c|c|}
\hline \multicolumn{2}{|r|}{ FUNCIONES } \\
\hline $\begin{array}{l}\text { Unidad de } \\
\text { Personal }\end{array}$ & $\begin{array}{l}\text { El diseño, la definición, coordinación } \\
\text { y verificación de políticas, } \\
\text { procesos y procedimientos en } \\
\text { lo relacionado con el talento } \\
\text { Humano en la Facultad. Integra } \\
\text { la gestión del talento humano con } \\
\text { el Plan Estratégico de la Faculta } \\
\text { de Ingeniería Industrial y con } \\
\text { actividades estratégicas de otras } \\
\text { dependencias. }\end{array}$ \\
\hline $\begin{array}{c}\text { Área de control y } \\
\text { permanencia }\end{array}$ & $\begin{array}{l}\text { Encargada de los procesos } \\
\text { relacionados con el control y } \\
\text { permanencia de los docentes } \\
\text { a dedicación exclusiva, tiempo } \\
\text { completo, tiempo parcial; así } \\
\text { como a los docentes contratados } \\
\text { por diversas modalidades, } \\
\text { en concordancia de su carga } \\
\text { académica; }\end{array}$ \\
\hline $\begin{array}{c}\text { Área } \\
\text { administrativa }\end{array}$ & $\begin{array}{l}\text { Encargada de los procesos } \\
\text { relacionados con la coordinación } \\
\text { y el establecimiento de normas y } \\
\text { procedimientos relacionados con la } \\
\text { nómina de los servidores públicos } \\
\text { de la Universidad, que incluye la } \\
\text { liquidación y pago de los salarios, } \\
\text { la seguridad social, prestaciones } \\
\text { sociales y bonos pensionales, entre } \\
\text { otros aspectos. } \\
\text { Para tal efecto, la división imparte } \\
\text { las directrices para planeación, } \\
\text { control, supervisión y evaluación. }\end{array}$ \\
\hline $\begin{array}{c}\text { Área de } \\
\text { capacitación }\end{array}$ & $\begin{array}{l}\text { Su misión es apoyar, participar e } \\
\text { influir activamente en la excelencia } \\
\text { de la Facultad de Ingeniería } \\
\text { Industrial a través de la Gestión del } \\
\text { talento Humano Docente, orientado } \\
\text { la ejecución de planes, programas } \\
\text { y proyectos en desarrollo de las } \\
\text { políticas institucionales establecidas } \\
\text { por la Unidad de Personal, en } \\
\text { materia de capacitación al personal. }\end{array}$ \\
\hline
\end{tabular}

El capital intelectual está constituido generalmente por tres componentes básicos y fuertemente interrelacionados: capital humano, capital estructural y capital relacional.
Composición de la Estructura Organizacional de la Facultad de Ingeniería Industrial

La fase de clasificación del personal administrativo y docente de la Facultad de Ingeniería Industrial de la UNMSMS se encuentra consignada en el CAPITULO X "DEL PERSONAL ADMINISTRATIVO Y DE SERVICIO" específicamente en los Art. $226^{\circ}$ "El personal administrativo y de servicio de la Universidad Nacional Mayor de San Marcos está conformado por los trabajadores que cumplen actividades administrativas, profesionales, técnicas o de servicio; que no son propias de la docencia y sirven de apoyo para el cumplimiento de los planes de la Universidad" así como del Art. $227^{\circ}$ que dice "El personal administrativo y de servicio de la Universidad está sujeto al régimen legal de los servidores públicos. Excepcionalmente habrá personal dedicado a labores de producción de bienes y/o servicios que se regirá por la legislación laboral respectiva Y DEL CAPÍTULO V - LOS PROFESORES; Art. 116 "Los profesores de la Universidad Nacional Mayor de San Marcos ejercen funciones de enseñanza-aprendizaje, investigación y proyección social. Es inherente a la función docente la capacitación permanente y la producción intelectual al servicio del desarrollo nacional y del progreso del país."

\section{Evaluación y Ratificación y Promoción docente}

La ratificación y promoción docente en la Universidad Nacional Mayor de San Marcos tienen como base legal: la Ley Universitaria $N^{\circ} 23733$ y sus modificaciones, la Ley $N^{\circ} 27444$ "Ley del Procedimiento Administrativo General", el Estatuto de la Universidad y el Decreto de Urgencia N ${ }^{\circ} 033-$ 2005.

RATIFICACIÓN DOCENTE: Con Resolución Rectoral N $01137-R-07$, de fecha 13 de marzo del 2007, se aprueba el "REGLAMENTO DE EVALUACIÓN PARA RATIFICACIÓN DOCENTE" que regula la evaluación académica de los docentes de la Universidad Nacional Mayor de San Marcos, para realizar su ratificación en las respectivas categorías.

\section{Bienestar Laboral y Capacitación}

En el Estatuto de la Universidad específicamente en el CAPÍTULO XI - DEL BIENESTAR UNIVERSITARIO se manifiesta en el Art. $241^{\circ}$ "La Universidad Nacional Mayor de San Marcos debe organizar programas de bienestar que contribuyan a crear condiciones adecuadas para el cumplimiento de sus fines, ofrecer a los profesores, estudiantes y trabajadores no docentes los servicios necesarios a fin de contribuir en mejorar su nivel socio-económico 
y el cumplimiento de sus obligaciones.." y en el Art. $246^{\circ}$ dice "... Las Facultades organizan Unidades de Bienestar, con la finalidad de cumplir sus propias actividades, las que coordinan con la Oficina de Bienestar de la Universidad, proporcionan la información que requiere dicha Oficina y formulan sugerencias sobre los programas establecidos y la creación de otros."; a su vez la Oficina de personal Plantear y ejecutar en coordinación con la Oficina General de Recursos Humanos,

programas de desarrollo y capacitación para el personal tal como manifiesta en el Manual de Organización y Funciones del 2011 item c, de las funciones generales del Jefe de Personal es "Plantear y ejecutar en coordinación con la Oficina General de Recursos Humanos, programas de desarrollo y capacitación para el personal."; donde se evidencia la importancia de la necesidad de capacitación y formación como insumo fundamental para la formulación del Plan de Capacitación 2013 $-2016$.

\section{Estímulos y distinciones}

Para las fases de implantación y compensación se hace cumplimiento de las políticas vinculadas al Plan Estratégico de la Facultad 2012 - 2021; Por el cual se determinan los lineamientos y el procedimiento para el otorgamiento de las distinciones y estímulos al personal administrativo y docente, esto concuerda con lo expresado por Calderón, Naranjo y Álvarez (2010) al referirse a los sistemas de información para bienestar y salud programa por el cual se agilizan los procesos de bienestar y se da trasparencia a su manejo, al igual que permite gestionar estratégicamente la política de remuneración integral logrando equidad interna y competitividad externa al tener un claro establecimiento de las normas y pasos para que el personal administrativo tenga sus correspondientes estímulos y distinciones; los estímulos permiten el reconocimiento de las actitudes y comportamientos de servicio hacia el cliente interno y externo y al trabajo en equipo.

\section{Fases del sistema de mejor gestión del talento humano}

En consecuencia el sistema de mejor gestión del talento humano en la facultad de Ingeniería Industrial, tiene como finalidad realizar mejora continua a través de acciones que garanticen y articulen las actividades propias de la GTH y permitan realizar una retroalimentación y revisión permanente para de sus procesos y por lo tanto del servicio a sus clientes internos y externos.
Es por ello que actualmente se tiene definido el macroproceso de GTH, y sus procesos, subprocesos y procedimientos se precisaron teniendo en cuenta los lineamientos y directrices generales de la gestión del talento humano, sus estatutos y normativas que lo soportan.

Como estrategia también se generó el manual especifico de funciones para los cargos contemplados en la plana administrativa-académica de la facultad, en donde se establece las funciones, competencias y grupos ocupacionales para los cargos de Carrera Administrativa.

En la siguiente tabla se presenta un análisis comparativo de los modelos analizados en esta investigación, en donde se definen el enfoque respecto a los componentes del capital intelectual esto es: capital humano, estructural y relacional; así como el objetivo o centro temático del modelo.

Selección de los modelos coincidentes de capital intelectual respecto a la Gestión del Talento Humano - GTH, en la facultad de Ingeniería de la UNMSM

Como se expuso en la parte metodológica uno de las técnicas para la recolección de la información fue la entrevista, en este caso, se realizó entrevista directa semi estructurada al Jefe de Personal de la facultad de ingeniería industrial, Lic. Ivar Farfan Muñoz, en donde se indago respecto a la situación del sistema de gestión del talento humano, cuales son los lineamientos, criterios, políticas y perspectivas que lo enmarcan.

* Existen oportunidades de desarrollo dentro de la carrera profesional y formación que se articulan con la dirección estratégica de la facultad de ingeniería industrial, evidencia de ello es la gestión de la plana administrativa.

* Modificación de la plana administrativa: como resultado de la cualificación del personal y de proceso de jubilación del personal de trámite documentario y el fallecimiento del jefe de la unidad de impresiones se realiza según las necesidades del servicio la modificación de la plana administrativa con el fin de articularla con la misión de la facultad.

* Las políticas de desarrollo profesional que se tiene en la facultad, responden a su articulación con el que hacer universitario y la actividad administrativa de apoyar la función misional, resultado de ello es el documento del plan de capacitación 2013-2016; que recoge las necesidades de capacitación de los diferentes cargos, dejando manifiesta cuales deben ser las habilidades claves que debe tener un funcionario administrativo en un plazo de 5 años. 


\begin{tabular}{|c|c|c|c|c|}
\hline $\begin{array}{l}\text { CAPITAL } \\
\text { INTELECTUAL }\end{array}$ & $\begin{array}{l}\text { CAPITAL } \\
\text { HUMANO }\end{array}$ & $\begin{array}{l}\text { CAPITAL } \\
\text { ESTRUCTURAL }\end{array}$ & $\begin{array}{c}\text { CAPITAL } \\
\text { RELACIONAL }\end{array}$ & OBJETIVO \\
\hline Skandia Navigator & Enfoque humano & $\begin{array}{c}\text { Clientes, } \\
\text { capacidad } \\
\text { organizativa, } \\
\text { innovación } \\
\text { y procesos }\end{array}$ & $\begin{array}{l}\text { Clientes y } \\
\text { mercado }\end{array}$ & $\begin{array}{l}\text { Hace visible el capital Intelectual } \\
\text { (no aparece en estado contable) }\end{array}$ \\
\hline Techology Broker & Activos humanos & $\begin{array}{c}\text { Activos de } \\
\text { propiedad } \\
\text { intelectual y } \\
\text { activos } \\
\text { de infraestructura }\end{array}$ & $\begin{array}{l}\text { Clientes y } \\
\text { mercado }\end{array}$ & $\begin{array}{l}\text { Metodología para auditar } \\
\text { información relacionada } \mathrm{Cl} \text {, } \\
\text { partiendo de ymdo } \mathrm{CT}+\mathrm{Cl}\end{array}$ \\
\hline $\begin{array}{l}\text { Canadian } \\
\text { Imperial Bank }\end{array}$ & Capital humano & Capital estructural & $\begin{array}{l}\text { Capital } \\
\text { clientes }\end{array}$ & $\begin{array}{l}\text { Estudiar relación entre } \mathrm{C} \text {. } \\
\text { Conocimiento } \mathrm{Cl} \text { y niveles de } \\
\text { aprendizaje. }\end{array}$ \\
\hline $\begin{array}{l}\text { Modelo Intelectual } \\
\text { de Dirección } \\
\text { Estratégica } \\
\text { por Competencia }\end{array}$ & Capital humano & Capital estructural & $\begin{array}{l}\text { Capital } \\
\text { relacional }\end{array}$ & $\begin{array}{l}\text { Profundiza capital intelectual } \\
\text { por competencia } C=\text { vir mdo - } \\
\text { vir. contables activos }\end{array}$ \\
\hline $\begin{array}{c}\text { Balanced } \\
\text { Scoreacard, } \\
\text { BSC o Cuadro de } \\
\text { Mando } \\
\text { Integral }\end{array}$ & Capital humano & $\begin{array}{l}\text { Clientes, } \\
\text { capacidad } \\
\text { organizativa, } \\
\text { innovación } \\
\text { y procesos }\end{array}$ & $\begin{array}{l}\text { Clientes y } \\
\text { mercado }\end{array}$ & $\begin{array}{l}\text { Medir resultados a través de la } \\
\text { Visión y a la estrategia }\end{array}$ \\
\hline $\begin{array}{c}\text { Modelo de } \\
\text { Intangible } \\
\text { Assets. Monitor el } \\
\text { Monitor de Activos } \\
\text { intangibles }\end{array}$ & Activos humanos & $\begin{array}{l}\text { Activos de } \\
\text { propiedad } \\
\text { intelectual }\end{array}$ & $\begin{array}{l}\text { Capital } \\
\text { relacional }\end{array}$ & $\begin{array}{l}\text { Estudia los intangibles como } \\
\text { generadores de flujos de caja } \\
\text { futuros. Vmdo. = Libros }+\mathrm{Cl}\end{array}$ \\
\hline $\begin{array}{l}\text { Modelo Western } \\
\text { Ontario }\end{array}$ & Capital humano & Capital estructural & $\begin{array}{l}\text { Capital } \\
\text { relacional }\end{array}$ & $\begin{array}{l}\text { Estudiar relaciones causa } \\
\text { efectos de elementos del } \mathrm{Cl} \text {, } \\
\text { y entre estos los resultados } \\
\text { empresariales }\end{array}$ \\
\hline
\end{tabular}

Fuente : Elaboración propia.

* Respecto al desempeño del personal administrativo se realizan las evaluaciones periódicas, sistemáticas, imparciales objetivas con la frecuencia que lo determine el reglamento. Fruto de ello se visualizan las debilidades y fortalezas del personal y se buscan acciones de mejora a través de mecanismos como capacitación, reinducción y sí es del caso ubicación.
* La gestión del conocimiento se está cristalizando a través del modelo SIGA, en donde la comunidad academia puede participar realizando sugerencias y mejoras a los procesos y procedimientos ya definidos, de tal manera que enriquezca y sea más eficiente y eficaz la operación.

* La GTH en la facultad de ingeniería industrial, se realiza teniendo en cuenta las funcione 
básicas de la administración del personal, más las estrategias propias de una entidad de educación, partiendo del plan estratégico, ley universitaria y estatutos de la universidad; así como los reglamentos de promoción y ratificación docente.

* Las coincidencias entre las perspectivas de los componentes de los diferentes modelos de Gestión del Capital Intelectual enfocado a la facultad, se analizaron luego de revisar la fundamentación teórica, los archivos documentales y los vértices centrales para la gestión en la facultad, contrastados respecto a los enfoques de los 7 modelos analizados en el marco teórico, encontrándose que los modelos más coincidentes son el modelo de CMI y el de competencias teniendo en cuenta lo siguiente:

* La Facultad de Ingeniería Industrial de la Universidad Nacional Mayor de San Marcos cuenta con instrumentos como el Plan Estratégico Global de Desarrollo, el sistema de Gestión Administrativa de la facultad de Ingeniería Industrial "SIGA", que se podría adaptar y ajustar como ensayo piloto al Cuadro de Mando Integral "CMI".

El objetivo del modelo de competencias es obtener una "competencia esencial" que está compuesta por tres elementos básicos distintos y que deben actuar adecuadamente, estos son: las personales (actitudes, aptitudes y habilidades); la tecnología (saber y experiencia acumulada), y las de la organización (procesos).

En la Facultad de Ingeniería Industrial se cuenta con estos tres elementos que permitirían alcanzar esa competencia esencial y que paralelo al modelo serian:

- Las personas: Personal administrativo calificado y comprometido

- Los procesos: EI SIGA y específicamente el SIGA de la GTH

- La tecnología: la experiencia y posicionamiento de la Facultad de Ingeniería Industrial en la UNMSM.

Así se plantea que a partir de proyectos de gestión que es posible construir un referente de política integrada del capital humano, a través de un plan de acción que contemple:

- El Plan de formación y desarrollo del personal cuenta con un plan de capacitación 2010-2012 que esa definido como "Conjunto coherente de acciones de capacitación y formación, que durante un periodo de tiempo y a partir de unos objetivos específicos, facilita el desarrollo de competencias, el mejoramiento de los procesos institucionales y el fortalecimiento de la capacidad laboral de los empleados a nivel individual y de equipo para conseguir los resultados y metas institucionales establecidas en una entidad".

- La gestión de las potencialidades de los empleados y los planes de carrera, situación que permite conectar, lo que quiere ser, lo que hace o sabe y lo que es capaz de ser; de tal manera que los perfiles y sus competencias individuales se conviertan en competencias colectivas y esenciales, gestionado el conocimiento y el capital intelectual. Otra herramienta importante ya construida en la Facultad, es el "Manual de Organización y Funciones para los cargos del personal administrativo"; que partir de información obtenida en las dependencias sobre los puestos de trabajo y contrastarlo frente a los procesos que se realizan en las mismas se verifica la misión y el objeto principal del puesto de trabajo.

Derivado de lo anterior y como estrategia de dirección y gestión del personal administrativo, es posible evidenciar las competencias personales "los conocimientos que un empleado necesita conocer, las destrezas que debe poseer con el fin de realizar su trabajo; las actitudes y motivaciones para desarrollar desde una perspectiva positiva los rasgos de una persona que están asociados con la efectividad en determinado trabajo; y las conductas y normas que una persona debe aceptar para con el fin de adaptarse al trabajo" (González. 2000); a partir de ello es posible entonces identificar las competencias básicas y diferenciales, las primeras corresponden a un desempeño básico para ejecutar un cargo y las diferenciales son las que con los mismos recursos y capacidades (competencias personales. Como fruto de ello las empresas pueden generar conocimiento e innovar en sus procesos, sus productos, en este caso facilita la visualización del desempeño del personal administrativo, identificando aquellos que con sus iniciativas y creatividad fortalecen el que hacer universitario.

\section{Lineamientos para implementar un modelo de gestión del capital intelectual en el personal administrativo en la Facultad de Ingeniería Industrial de la UNMSMS}

Para familiarizarnos con los términos en el Cuadro De Mando Integral se indica siguientes componentes en el escenario Universitario 
En este sentido, Cañibano et al. (2000, p. 170) señala "la utilidad del CMI reside en que permite a los gerentes controlar simultáneamente los resultados financieros en el corto plazo y el progreso de la adquisición de los intangibles que aseguran el éxito de la empresa".

Es importante traer a colación que el SIGA, dentro de sus objetivos vigentes menciona se pueden "Fortalecer continuamente el desarrollo institucional mediante el mejoramiento continuo de sus procesos académicos y administrativos y el aprendizaje de la organización en un marco multisedes"; con referencia a la anterior se propone estos lineamientos del cuadro de Mando integral y el modelo estrategia por competencia, lo cual aporta pasos para su adopción en la dirección de personal administrativo docente. Con el fin de suscitar y mejorar el servicio que presta esta dependencia y elevar el grado de satisfacción del personal.

\section{Paso N. 1: Autoevaluación}

Como toda propuesta de mejora, es necesario hacer un diagnóstico del departamento en general, de acuerdo a las metas propuestas del plan de desarrollo institucional, donde puedan observarse: Misión, visión, formación de administrativos, dedicación, optimización de procesos, formación integral y desarrollo humano, cambio organizacional, apoyándose de técnicas sencillas como la matriz Fortalezas, Debilidades, Amenazas y Oportunidades "FODA"

\section{Paso N. ${ }^{\circ}$ 2: Mapa Estratégico del CMI}

El mapa estratégico tiene como finalidad recoger en una visión ampliada donde no se puede perder de vista la relación que debe existir entre cada estrategia y línea de acción para la consecución de los objetivos.

Paso N. $^{\circ}$ 3. Se elabora el mapa estratégico y las relaciones causa-efecto, y las relaciones clave para articular la estrategia.

\section{CONCLUSIONES Y RECOMENDACIONES}

\subsection{Conclusiones}

Teniendo en cuenta los resultados de la presente investigación las conclusiones más importantes son:

- Se demuestran las hipótesis planteadas en el capítulo III con los resultados presentados en el capítulo $\mathrm{V}$.
PERSPECTIVA DEL CLIENTE-USUARIO

\begin{tabular}{|l|l|}
\hline \multicolumn{1}{|c|}{ Objetivos } & \multicolumn{1}{c|}{ Indicadores } \\
\hline $\begin{array}{l}\text { Acrecentar el grado } \\
\text { satisfacción nuestros } \\
\text { Clientes. }\end{array}$ & $\begin{array}{l}N^{\circ} \text { de actuaciones } \\
\text { realizadas } \\
\text { - Grado satisfacción } \\
\text { usuarios } \\
-N^{\circ} \text { administrativos } \\
\text { participantes en la } \\
\text { actividad }\end{array}$ \\
\hline $\begin{array}{l}\text { Diseñar un modelo de evaluación del grado } \\
\text { de satisfacción de nuestros clientes y del } \\
\text { cumplimiento de sus expectativas. }\end{array}$ \\
\hline
\end{tabular}

PERSPECTIVA DE APRENDIZAJE Y CRECIMIENTO

\begin{tabular}{|l|l|}
\hline \multicolumn{1}{|c|}{ Objetivos } & \multicolumn{1}{c|}{ Indicadores } \\
\hline $\begin{array}{l}\text { Entregar a la } \\
\text { Universidad los } \\
\text { mejores recursos } \\
\text { para la docencia, } \\
\text { la investigación y la } \\
\text { prestación de servicios. }\end{array}$ & $\begin{array}{l}N^{0} \text { PC's nuevos y/o } \\
\text { sustituidos } \\
\text { - Obtención } \\
\text { Certificación } \\
\text { Equipo de alta } \\
\text { tecnología. } \\
\text { Desarrollar las } \\
\text { capacidades de las } \\
\text { personas } \\
\text { Carga/capacidad por Á }\end{array}$ \\
\hline $\begin{array}{l}\text { Desarrollar } \\
\text { capacidades de las } \\
\text { personas }\end{array}$ & $\begin{array}{l}\text { Carga/capacidad por } \\
\text { Área } \\
\text { No horas formación } \\
\text { / horas dedicadas al } \\
\text { trabajo }\end{array}$ \\
\hline
\end{tabular}

Fuente: Elaboración propia

- Al realizar una revisión teórica de los modelos de gestión de capital intelectual, se encontró que bajo la actual dinámica de un entorno económico globalizado y de conocimiento el capital humano es hoy una de las palancas dinamizadoras de la económica a través de la innovación y el conocimiento.

- Los enfoques tradicionales que privilegian la racionalidad económica y la estrategia sin estar 
integrados a una naturaleza de conocimiento y de generación de valor están inmersos en un proceso de gestión del siglo pasado, por tanto adoptar modelos o enfoques que privilegien los activos intangibles redundaran en beneficios y pasaran de ser competidores a tener competencias esenciales para su operación.

- En la facultad de ingeniería industrial se encontró que no se tiene implementado un modelo específico respecto a los referentes teóricos para la gestión de capital intelectual del personal administrativo-docente; sin embargo sí se cuenta con un horizonte dado por un documento guía: líneas y directrices generales para la gestión del talento humano.

- Al proponer unos lineamientos para implementar un modelo de gestión del capital intelectual en el personal administrativo en la facultad de ingeniería industrial, basados en la propuesta presentada donde se fusionan los modelos de $\mathrm{CMl}$ y de dirección estratégica por competencias se tiene como ventaja la alineación de los objetivos del personal administrativo con la visión universitaria donde se comparten, interiorizan, motivan y orientan los logros por objetivos comunes.

- La experiencia adquirida a través del estudio de caso realizado en el presente trabajo permite tener una base explicativa de cómo las IES a través de su principal función la cuales la generación del conocimiento, pueden aportar al interior de su estrategia la valoración de los intangibles cuando se ajustan o adoptan modelos de gestión del capital intelectual en el personal administrativo, socio estratégico de las tres funciones misionales de las universidades, es decir la investigación, la extensión y la docencia.

\subsection{Recomendaciones}

- La concepción de la calidad, la eficiencia y la eficacia debe estar presente en la gestión, por esta razón el nuevo reto de las universidades es valorar de manera integral su capital intelectual, por lo que se recomienda realizar un proceso de revisión de cómo se gestiona el capital humano específicamente el personal administrativo, dado que en este tipo de instituciones aunque se reconoce su valor y la labor que realiza, queda en un segundo plano frente a la gestión del personal docente.
- Adaptar un modelo de capital intelectual, permitirá entonces realizar conexiones reales entre la operación y la estrategia, para ello es indispensable que se provea de recursos humanos y financieros para su ejecución.

- Es necesario realizar una valoración de las implicaciones de un modelo de gestión de capital intelectual, cuando se toma como referente el cuadro de mando integral y el direccionamiento estratégico por competencias, dado que de los mismos es posible que se desprenda un sistema de retribución variable coherente con la estrategia y que se da por la diferencia en las competencias básicas (desempeño básico) y las competencias diferenciadores (desempeño superior).

\section{REFERENCIAS BIBLIOGRÁFICAS}

[1] Bernárdez M. (2008). Capital Intelectual: Creación De Valor En La Sociedad Del Conocimiento. USA: Autor House

[2] Brookin, A. (2010). Intellectual Capital. Cengage Learning .

[3] Bueno Campos, E. (1999). Gestión del Conocimiento, Aprendizaje y Capital Intelectual. Boletín del club Intelect Nro 01.

[4] Bueno Campos, E. (2003). Gestión del Conocimiento en Universidades y Organismos Públicos de Investigación. Recuperado el 15 de 10 de 2013, de http://www.madrimasd.org/ informacionidi/biblioteca/publicacion/doc/16 GestionConocimientoUniversidadesOPIS.pdf

[5] Carrión J. (2007). Estrategia: de la visión a la acción. Madrid: ESIC

[6] Comunidad de Marid (2002). Capital Intelectual y Producción Científica. Madrid: Consejería de Educación.

[7] Diaz Muñante, J. R. (2007). Modelo de Gestión del Conocimiento (GC)Aplicado a la Universidad Pública en el Perú. Lima.

[8] Edvisson L. et al (2003). El Capital Intelectual: Cómo Identificar y Calcular el Valor de Los Recursos. España: Gestión 2000

[9] Fuentes Morales, B. (2009). La Gestión de Conocimiento en las Relaciones Académico Empresariales un Nuevo Enfoque para Analizar el Impacto del Conocimiento Académico. Recuperado el 18 de 10 de 2013, de http:// riunet.upv.es/bitstream/handle/10251/8334/ tesisUPV3275.pdf?sequence $=1$ 
[10] García, Z. T. (2010). Diseño de un modelo multifactorial y dinámico (MFD) para la medición del capital intelectual de las empresas de manufactura del Perú. Lima.

[11] Hernández, Fernandez \& Baptista. (2010). Metodología de la investigación. México: McGraw-Hill.

[12] Inche Mitma, J. (2010). Modelo dinámico de gestión del conocimiento basado en el aprendizaje organizacional en una institución educativa en el Perú. Lima.

[13] Mantilla S. (2004). Capital Intelectual \& Contabilidad del Conocimiento. Bogotá: Ecoe Ediciones.

[14] Mosquera Cardona, L. E. (2011). Gestión del Capital Intelectual de las Instituciones de Educación Superior. Colombia.

[15] Ramírez Córcoles, Y. (s.f.). Cómo Gestionar el Capital Intelectual en las Universidades
Públicas Españolas: El Cuadro de Mando Integral. Recuperado el 7 de 10 de 2013, de http://www.observatorio-iberoamericano.org/ RICG/No_16/Yolanda_Ramirez_Corcoles.pdf

[16] Riesco M. (2006). El Negocio es el Conocimiento. España: Díaz de Santos

[17] Roos J. et al (2001). Capital Intelectual: EI Valor Intangible de la Empresa. España: Paidós Ibérica

[18] Sánchez H. y Reyes C. (2009). Metodología y Diseños en la Investigación Científica. Lima: Visión Universitaria.

[19] Tafur, R. (1995). La Tesis Universitaria. Lima: Mantaro

[20] Von Roth, A. (2008). Gestión del Conocimiento y la Competitividad en los 90. http://www.uch. edu.ar/rrhh. 